5-1-2015

\title{
Test for the Equality of Partial Correlation Coefficients for Two Populations
}

\author{
Madhusudan Bhandary \\ Columbus State University, bhandary_madhusudan@columbusstate.edu \\ Arjun K. Gupta \\ Bowling Green State University, gupta@bgnet.bgsu.edu
}

Follow this and additional works at: http:// digitalcommons.wayne.edu/jmasm

Part of the Applied Statistics Commons, Social and Behavioral Sciences Commons, and the Statistical Theory Commons

\section{Recommended Citation}

Bhandary, Madhusudan and Gupta, Arjun K. (2015) "Test for the Equality of Partial Correlation Coefficients for Two Populations," Journal of Modern Applied Statistical Methods: Vol. 14 : Iss. 1 , Article 10. DOI: $10.22237 /$ jmasm/1430453340

Available at: http://digitalcommons.wayne.edu/jmasm/vol14/iss1/10

This Regular Article is brought to you for free and open access by the Open Access Journals at DigitalCommons@WayneState. It has been accepted for inclusion in Journal of Modern Applied Statistical Methods by an authorized editor of DigitalCommons@WayneState. 


\section{Test for the Equality of Partial Correlation Coefficients for Two Populations}

\section{Cover Page Footnote}

Authors would like to thank Mr. Mohammad Aziz for computational help. 


\title{
Test for the Equality of Partial Correlation Coefficients for Two Populations
}

\author{
Madhusudan Bhandary \\ Columbus State University \\ Columbus, GA
}

\author{
Arjun K. Gupta \\ Bowling Green State University \\ Bowling Green, $\mathrm{OH}$
}

\begin{abstract}
A likelihood ratio test for the equality of two partial correlation coefficients based on two independent multinormal samples has been derived. The large sample Z-test for the same problem has also been discussed. The power analysis of the two tests is obtained. It has been found that the approximate likelihood ratio (ALR) test showed consistently better results than $\mathrm{Z}$-test in terms of power. The size of the ALR test is slightly more than the alpha level. The ALR test is recommended strongly for use in practice.
\end{abstract}

Keywords: $\quad$ Likelihood ratio test, partial correlation coefficients, asymptotic distribution

\section{Introduction}

The partial correlation coefficient is frequently used to measure the correlation of two variables after eliminating the effect of other variable(s) in a set of correlated variables. For example, it may be of interest to know the correlation between intelligence and weight of people after eliminating the effect of age. In this case, the partial correlation coefficient will give the appropriate measure of the required correlation.

Statistical inference concerning the partial correlation coefficient for a single sample problem has been studied by Fisher (1924). Some discussions are also given in Anderson (2003). Surprisingly, the extension of inference problem concerning partial correlation coefficient to two-sample as well as multi-sample problems has received very little attention. Test for the equality of several multiple and partial correlation coefficients based on several independent Wishart densities has been derived by Gupta and Kabe (2001).

Dr. Bhandary is a Professor of Mathematics. Email him at:

bhandary_madhusudan@columbusstate.edu,Dr. Gupta is a Distinguished Professor in the Department of Mathematics and Statistics. Email him at: gupta@bgnet.bgsu.edu. 


\section{BHANDARY \& GUPTA}

In this paper, it has been considered the problem of testing the equality of two partial correlation coefficients based on two independent multinormal samples. It could be of interest to see whether the partial correlation of intelligence and weight of children after eliminating the effect of age in United States differs from the same in Asia and therefore, it is needed to develop test for the equality of partial correlation coefficients.

In the next section, the likelihood ratio test for the equality of two partial correlation coefficients is derived and the large sample test is also discussed. The power analysis of the tests is obtained through simulation and is discussed in the final section.

It has been found that the approximate likelihood ratio (ALR) test shows consistently better results than Z-test in terms of power. The size of the ALR test is slightly more than the alpha level. The ALR test is recommended strongly for use in practice.

\section{Test of $H_{0}: \rho_{12.3}^{(1)}=\rho_{12.3}^{(2)}$ Versus $\boldsymbol{H}_{1}: \rho_{12.3}^{(1)} \neq \rho_{12.3}^{(2)}$}

\section{Likelihood ratio test}

Let $\underset{\sim}{x}=\left(x_{1} \ldots x_{p}\right)^{\prime}$ be a $p \times 1$ vector of observations and $\underset{\sim}{\mu}=\left(\mu_{1} \ldots \mu_{p}\right)^{\prime}$ be a $p \times 1$ vector of unknown means. It is assumed that $\underset{\sim}{x} \sim N_{p}(\underset{\sim}{\mu}, \Sigma)$, where $\Sigma$ is a $p \times p$ unknown positive definite matrix and $N_{p}$ denotes $p$-variate normal distribution. It will be considered the case of $p=3$ in this article, i.e.

$$
\left.\underset{\sim}{x}=\left(\begin{array}{lll}
x_{1} & x_{2} & x_{3}
\end{array}\right)^{\prime} \sim N_{3}\left(\begin{array}{lll}
\mu_{1} & \mu_{2} & \mu_{3}
\end{array}\right)^{\prime},\left(\begin{array}{lll}
\sigma_{11} & \sigma_{12} & \sigma_{13} \\
\sigma_{21} & \sigma_{22} & \sigma_{23} \\
\sigma_{31} & \sigma_{32} & \sigma_{33}
\end{array}\right)\right)
$$

It follows from (1) that

$$
\left(\begin{array}{l}
x_{1.3} \\
x_{2.3}
\end{array}\right) \sim N_{2}\left(\left(\begin{array}{l}
\mu_{1.3} \\
\mu_{2.3}
\end{array}\right),\left(\begin{array}{cc}
\sigma_{11.3} & \rho_{12.3} \sqrt{\sigma_{11.3}} \sqrt{\sigma_{22.3}} \\
\rho_{12.3} \sqrt{\sigma_{11.3}} \sqrt{\sigma_{22.3}} & \sigma_{22.3}
\end{array}\right)\right)
$$


where,

$$
\begin{aligned}
\left(\begin{array}{l}
x_{1.3} \\
x_{2.3}
\end{array}\right) & =\left(\begin{array}{l}
x_{1} \\
x_{2}
\end{array}\right) \text { given } X_{3}=x_{3} \\
\mu_{1.3} & =\mu_{1}+\frac{\sigma_{13}}{\sigma_{33}}\left(x_{3}-\mu_{3}\right) \\
\mu_{2.3} & =\mu_{2}+\frac{\sigma_{23}}{\sigma_{33}}\left(x_{3}-\mu_{3}\right) \text { and } \\
\rho_{12.3} & =\frac{\sigma_{12.3}}{\sqrt{\sigma_{11.3}} \sqrt{\sigma_{22.3}}}=\text { partial correlation coefficient }
\end{aligned}
$$

between $X_{1}$ and $X_{2}$ given $X_{3}=x_{3}$.

Now, the joint probability density function of $x_{1.3}$ and $x_{2.3}$ is given by

$$
\begin{aligned}
& f\left(x_{1.3}, x_{2.3} ; \theta\right)= \\
& \frac{1}{2 \pi \sqrt{\sigma_{11.3} \sigma_{22.3}\left(1-\rho_{12.3}^{2}\right)}} e^{-\frac{1}{2\left(1-\rho_{12.3}^{2}\right)}\left[\frac{\left(x_{1.3}-\mu_{1.3}\right)^{2}}{\sigma_{11.3}}-2 \rho_{12.3}\right.} \frac{\left(x_{1.3}-\mu_{1.3}\right)}{\sqrt{\sigma_{11.3}}} \frac{\left(x_{2.3}-\mu_{2.3}\right)}{\left.\sqrt{\sigma_{22.3}}+\frac{\left(x_{2.3}-\mu_{2.3}\right)^{2}}{\sigma_{22.3}}\right]}
\end{aligned}
$$

where, $\theta$ denotes the parameter vector of the distribution. Let $X_{\sim}^{(1)}, X_{\sim}^{(2)}, \ldots,{\underset{\sim}{X}}^{(n)}$ be $3 \times 1$ vector of observations i.i.d. $\sim N_{3}(\underset{\sim}{\mu}, \Sigma)$, where $\underset{\sim}{\mu}$ and $\Sigma$ are given by (1).

From (2), it can be said that

$$
\left(\begin{array}{l}
x_{1.3}^{(i)} \\
x_{2.3}^{(i)}
\end{array}\right) \sim N_{2}\left(\left(\begin{array}{l}
\mu_{1.3} \\
\mu_{2.3}
\end{array}\right),\left(\begin{array}{cc}
\sigma_{11.3} & \rho_{12.3} \sqrt{\sigma_{11.3}} \sqrt{\sigma_{22.3}} \\
\rho_{12.3} \sqrt{\sigma_{11.3}} \sqrt{\sigma_{22.3}} & \sigma_{22.3}
\end{array}\right)\right)
$$

and $\left(\begin{array}{l}x_{1.3}^{(i)} \\ x_{2.3}^{(i)}\end{array}\right)$ 's are independent, $i=1,2, \ldots, n$. Using (3), the likelihood function of $\left(\begin{array}{c}x_{1.3}^{(i)} \\ x_{2.3}^{(i)}\end{array}\right), i=1,2, \ldots, n$ is obtained as follows: 


\section{BHANDARY \& GUPTA}

$$
\begin{aligned}
& L\left(x_{1.3}^{(i)}, x_{2.3}^{(i)}, i=1, \ldots, n ; \theta\right)= \\
& \frac{1}{\left(2 \pi \sqrt{\sigma_{11.3} \sigma_{22.3}\left(1-\rho_{12.3}^{2}\right)}\right)^{n}} e^{-\frac{1}{2\left(1-\rho_{12.3}^{2}\right)}\left[\sum_{i=1}^{n} \frac{\left(x_{1.3}^{(i)}-\mu_{1.3}\right)^{2}}{\sigma_{11.3}}-2 \rho_{12.3} \sum_{i=1}^{n} \frac{\left(x_{1.3}^{(i)}-\mu_{1.3}\right)}{\sqrt{\sigma_{11.3}}} \frac{\left(x_{i .3}^{(i)}-\mu_{2.3}\right)}{\sqrt{\sigma_{22.3}}}+\sum_{i=1}^{n} \frac{\left(x_{2.3}^{(i)}-\mu_{2.3}\right)^{2}}{\sigma_{22.3}}\right]}
\end{aligned}
$$

Now, it is considered two sample problem with $n_{1}$ and $n_{2}$ observations respectively from each population. Let $\underset{\sim}{x^{(i)}}=\left(\begin{array}{lll}x_{1}^{(i)} & x_{2}^{(i)} & x_{3}^{(i)}\end{array}\right)^{\prime}$ be the $i^{\text {th }}$

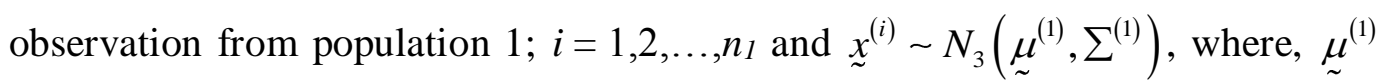
and $\Sigma^{(1)}$ are the mean vector and dispersion matrix respectively and hence

$$
\begin{aligned}
& \left(\begin{array}{l}
x_{1.3}^{(i)} \\
x_{2.3}^{(i)}
\end{array}\right) \sim N_{2}\left(\left(\begin{array}{l}
\mu_{1.3}^{(i)} \\
\mu_{2.3}^{(i)}
\end{array}\right),\left(\begin{array}{cc}
\sigma_{11.3}^{(1)} & \rho_{12.3}^{(1)} \sqrt{\sigma_{11.3}^{(1)}} \sqrt{\sigma_{22.3}^{(1)}} \\
\rho_{12.3}^{(1)} \sqrt{\sigma_{11.3}^{(1)}} \sqrt{\sigma_{22.3}^{(1)}} & \sigma_{22.3}^{(1)}
\end{array}\right), i=1,2, \ldots, n_{1} \quad\right. \text { where, } \\
& \left(\begin{array}{l}
x_{1.3}^{(i)} \\
x_{2.3}^{(i)}
\end{array}\right)=\left(\begin{array}{c}
x_{1}^{(i)} \\
x_{2}^{(i)}
\end{array}\right) \text { given } X_{3}^{(i)}=x_{3}^{(i)}
\end{aligned}
$$

Let $z^{(j)}=\left(\begin{array}{lll}z_{1}^{(j)} & z_{2}^{(j)} & z_{3}^{(j)}\end{array}\right)^{\prime} \quad$ be the $j^{\text {th }}$ observation from population $2 ; j=1,2, \ldots, n_{2}$ and $z_{\sim}^{(j)} \sim N_{3}\left(\underset{\sim}{\mu}(2), \Sigma^{(2)}\right)$, where, $\underset{\sim}{\underset{\sim}{(2)}}$ and $\Sigma^{(2)}$ are the mean vector and dispersion matrix respectively and hence $\left(\begin{array}{l}z_{1.3}^{(j)} \\ z_{2.3}^{(j)}\end{array}\right) \sim N_{2}\left(\left(\begin{array}{l}\mu_{1.3}^{(2)} \\ \mu_{2.3}^{(2)}\end{array}\right),\left(\begin{array}{cc}\sigma_{11.3}^{(2)} & \rho_{12.3}^{(2)} \sqrt{\sigma_{11.3}^{(2)}} \sqrt{\sigma_{22.3}^{(2)}} \\ \rho_{12.3}^{(2)} \sqrt{\sigma_{11.3}^{(2)}} \sqrt{\sigma_{22.3}^{(2)}} & \sigma_{22.3}^{(2)}\end{array}\right)\right), \quad$ where $\left(\begin{array}{l}z_{1.3}^{(j)} \\ z_{2.3}^{(j)}\end{array}\right)=\left(\begin{array}{l}z_{1}^{(j)} \\ z_{2}^{(j)}\end{array}\right)$ given $Z_{3}^{(j)}=z_{3}^{(j)}$. Under the above setup, the likelihood ratio test for testing $H_{0}$ Vs. $H_{1}$ is derived as follows: Under $H_{1}$, the log-likelihood function based on $\left(\begin{array}{l}x_{1.3}^{(i)} \\ x_{2.3}^{(i)}\end{array}\right), i=1,2, \ldots, n_{1}$ and $\left(\begin{array}{l}z_{1.3}^{(j)} \\ z_{2.3}^{(j)}\end{array}\right), j=1,2, \ldots, n_{2}$ is 


$$
\begin{aligned}
& \log L_{1}=-\left(n_{1}+n_{2}\right) \log 2 \pi-\frac{n_{1}}{2}\left\{\log \sigma_{11.3}^{(1)}+\log \sigma_{22.3}^{(1)}+\log \left(1-\rho_{12.3}^{(1) 2}\right)\right\} \\
& -\frac{n_{2}}{2}\left\{\log \sigma_{11.3}^{(2)}+\log \sigma_{22.3}^{(2)}+\log \left(1-\rho_{12.3}^{(2) 2}\right)\right\} \\
& -\frac{1}{2\left(1-\rho_{12.3}^{(1) 2}\right)}\left[\begin{array}{l}
\left.\sum_{i=1}^{n_{1}} \frac{\left(x_{1.3}^{(i)}-\mu_{1.3}^{(1)}\right)^{2}}{\sigma_{11.3}^{(1)}}-2 \rho_{12.3}^{(1)} \sum_{i=1}^{n_{1}} \frac{\left(x_{1.3}^{(i)}-\mu_{1.3}^{(1)}\right)}{\sqrt{\sigma_{11.3}^{(1)}}} \frac{\left(x_{2.3}^{(i)}-\mu_{2.3}^{(1)}\right)}{\sqrt{\sigma_{22.3}^{(1)}}}+\right] \\
\sum_{i=1}^{n_{1}} \frac{\left(x_{2.3}^{(i)}-\mu_{2.3}^{(1)}\right)^{2}}{\sigma_{22.3}^{(1)}}
\end{array}\right] \\
& -\frac{1}{2\left(1-\rho_{12.3}^{(2) 2}\right)}\left[\begin{array}{l}
\sum_{j=1}^{n_{2}} \frac{\left(z_{1.3}^{(j)}-\mu_{1.3}^{(2)}\right)^{2}}{\sigma_{11.3}^{(2)}}-2 \rho_{12.3}^{(2)} \sum_{j=1}^{n_{2}} \frac{\left(z_{1.3}^{(j)}-\mu_{1.3}^{(2)}\right)}{\sqrt{\sigma_{11.3}^{(2)}}} \frac{\left(z_{2.3}^{(j)}-\mu_{2.3}^{(2)}\right)}{\sqrt{\sigma_{22.3}^{(2)}}}+ \\
\sum_{j=1}^{n_{1}} \frac{\left(z_{2.3}^{(j)}-\mu_{2.3}^{(2)}\right)^{2}}{\sigma_{22.3}^{(2)}}
\end{array}\right]
\end{aligned}
$$

Maximizing $\log L_{1}$ in (6) w.r.t. $\mu_{1.3}^{(r)}, \mu_{2.3}^{(s)}, \sigma_{11.3}^{(r)}, \sigma_{22.3}^{(s)}, \rho_{12.3}^{(r)} ; r, s=1,2$, it can be obtained that

$$
\operatorname{SupL}_{H_{1}}=\frac{1}{(2 \pi)^{n_{1}+n_{2}}\left(\frac{a_{11.3}}{n_{1}}\right)^{\frac{n_{1}}{2}}\left(\frac{a_{22.3}}{n_{1}}\right)^{\frac{n_{2}}{2}}\left(1-\hat{\rho}_{12.3}^{(1) 2)}\right)^{\frac{n_{1}}{2}}\left(\frac{b_{11.3}}{n_{2}}\right)^{\frac{n_{2}}{2}}\left(\frac{b_{22.3}}{n_{2}}\right)^{\frac{n_{2}}{2}}\left(1-\hat{\rho}_{12.3}^{(2) 2}\right)^{\frac{n_{2}}{2}}} \cdot e^{-\left(n_{1}+n_{2}\right)}(7)
$$

where,

$$
\begin{aligned}
a_{\alpha \beta .3} & =\sum_{i=1}^{n_{1}}\left(x_{\alpha .3}^{(i)}-\bar{x}_{\alpha .3}\right)\left(x_{\beta .3}^{(i)}-\bar{x}_{\beta .3}\right) \\
\bar{x}_{\alpha .3} & =\frac{1}{n_{1}} \sum_{i=1}^{n_{1}} x_{\alpha .3}^{(i)} ; \alpha, \beta=1,2 \\
b_{m n .3} & =\sum_{j=1}^{n_{2}}\left(z_{m .3}^{(j)}-\bar{z}_{m .3}\right)\left(z_{n .3}^{(j)}-\bar{z}_{n .3}\right) \\
\bar{z}_{m .3} & =\frac{1}{n_{2}} \sum_{j=1}^{n_{2}} z_{m .3}^{(j)} ; m, n=1,2
\end{aligned}
$$




\section{BHANDARY \& GUPTA}

and $\hat{\rho}_{12.3}^{(1)}=\frac{a_{12.3}}{\sqrt{a_{11.3} a_{22.3}}}, \hat{\rho}_{12.3}^{(2)}=\frac{b_{12.3}}{\sqrt{b_{11.3} b_{22.3}}}$.

Similarly, under $H_{0}$, the log-likelihood function is given by

$$
\begin{aligned}
& \log L_{0}=-\left(n_{1}+n_{2}\right) \log 2 \pi-\frac{n_{1}}{2}\left\{\log \sigma_{11.3}^{(1)}+\log \sigma_{22.3}^{(1)}+\log \left(1-\rho^{2}\right\}\right. \\
& -\frac{n_{2}}{2}\left\{\log \sigma_{11.3}^{(2)}+\log \sigma_{22.3}^{(2)}+\log \left(1-\rho^{2}\right\}\right. \\
& -\frac{1}{2\left(1-\rho^{2}\right)}\left[\begin{array}{l}
\left.\sum_{i=1}^{n_{1}} \frac{\left(x_{1.3}^{(i)}-\mu_{1.3}^{(1)}\right)^{2}}{\sigma_{11.3}^{(1)}}-2 \rho \sum_{i=1}^{n_{1}} \frac{\left(x_{1.3}^{(i)}-\mu_{1.3}^{(1)}\right)}{\sqrt{\sigma_{11.3}^{(1)}}} \frac{\left(x_{2.3}^{(i)}-\mu_{2.3}^{(1)}\right)}{\sqrt{\sigma_{22.3}^{(1)}}}+\right] \\
\sum_{i=1}^{n_{1}} \frac{\left(x_{2.3}^{(i)}-\mu_{2.3}^{(1)}\right)^{2}}{\sigma_{22.3}^{(1)}}
\end{array}\right] \\
& -\frac{1}{2\left(1-\rho^{2}\right)}\left[\begin{array}{l}
\left.\sum_{j=1}^{n_{2}} \frac{\left(z_{1.3}^{(j)}-\mu_{1.3}^{(2)}\right)^{2}}{\sigma_{11.3}^{(2)}}-2 \rho \sum_{j=1}^{n_{2}} \frac{\left(z_{1.3}^{(j)}-\mu_{1.3}^{(2)}\right)}{\sqrt{\sigma_{11.3}^{(2)}}} \frac{\left(z_{2.3}^{(j)}-\mu_{2.3}^{(2)}\right)}{\sqrt{\sigma_{22.3}^{(2)}}}+\right] \\
\sum_{j=1}^{n_{2}} \frac{\left(z_{2.3}^{(j)}-\mu_{2.3}^{(2)}\right)^{2}}{\sigma_{22.3}^{(2)}}
\end{array}\right]
\end{aligned}
$$

where, $\rho=$ common value of $\rho_{12.3}^{(1)}$ and $\rho_{12.3}^{(2)}$ under $H_{0}$. Maximizing $\log L_{0}$ in (8) w.r.t. $\mu_{1.3}^{(1)}, \mu_{2.3}^{(1)}, \mu_{1.3}^{(2)}, \mu_{2.3}^{(2)}$ it is obtained that

$\hat{\mu}_{1.3}^{(1)}=\bar{x}_{1.3}, \hat{\mu}_{2.3}^{(1)}=\bar{x}_{2.3}, \hat{\mu}_{1.3}^{(2)}=\bar{z}_{1.3}, \hat{\mu}_{2.3}^{(2)}=\bar{z}_{2.3}$

Now, $\frac{\partial \log L_{0}}{\partial \sigma_{11.3}^{(1)}}=0 \Rightarrow-\frac{n_{1}}{\sigma_{11.3}^{(1)}}+\frac{1}{\left(1-\rho^{2}\right)}\left\{\frac{a_{11.3}}{\sigma_{11.3}^{(1) 2}}-\frac{\rho a_{12.3}}{\sigma_{11.3}^{(1) 3 / 2} \sigma_{22.3}^{(1) 1 / 2}}\right\}=0$

$$
\text { i.e. } \frac{a_{11.3}}{\sigma_{11.3}^{(1)}}-\frac{\rho a_{12.3}}{\sqrt{\sigma_{11.3}^{(1)}} \sqrt{\sigma_{22.3}^{(1)}}}=n_{1}\left(1-\rho^{2}\right)
$$

Similarly, 


$$
\frac{\partial \log L_{0}}{\partial \sigma_{22.3}^{(1)}}=0 \Rightarrow \frac{a_{22.3}}{\sigma_{22.3}^{(1)}}-\frac{\rho a_{12.3}}{\sqrt{\sigma_{11.3}^{(1)}} \sqrt{\sigma_{22.3}^{(1)}}}=n_{1}\left(1-\rho^{2}\right)
$$

It is obtained by adding the equations (9) and (10) that

$$
\frac{a_{11.3}}{\hat{\sigma}_{11.3}^{(1)}}+\frac{a_{22.3}}{\hat{\sigma}_{22.3}^{(1)}}-\frac{2 \rho a_{12.3}}{\sqrt{\hat{\sigma}_{11.3}^{(1)}} \sqrt{\hat{\sigma}_{22.3}^{(1)}}}=2 n_{1}\left(1-\rho^{2}\right) .
$$

From (9) and (10), it follows that

$$
\frac{a_{11.3}}{\hat{\sigma}_{11.3}^{(1)}}=\frac{a_{22.3}}{\hat{\sigma}_{22.3}^{(1)}}=k(\text { say })
$$

From (11) and (12), it can be obtained that $2 k-2 \rho \hat{\rho}_{12.3}^{(1)} k=2 n_{1}\left(1-\rho^{2}\right)$

$$
\text { i.e. } k=\frac{n_{1}\left(1-\rho^{2}\right)}{1-\rho \hat{\rho}_{12.3}^{(1)}}
$$

Similarly,

$$
k^{*}=\frac{n_{1}\left(1-\rho^{2}\right)}{1-\rho \hat{\rho}_{12.3}^{(2)}} \text { where, } k^{*}=\frac{b_{11.3}}{\hat{\sigma}_{11.3}^{(2)}}=\frac{b_{22.3}}{\hat{\sigma}_{22.3}^{(2)}}
$$

From (12), and (14) it follows that

$$
\begin{aligned}
& \hat{\sigma}_{11.3}^{(1)}=\frac{a_{11.3}}{k}, \hat{\sigma}_{22.3}^{(1)}=\frac{a_{22.3}}{k}, \hat{\sigma}_{11.3}^{(2)}=\frac{b_{11.3}}{k^{*}}, \text { and } \hat{\sigma}_{22.3}^{(2)}=\frac{b_{22.3}}{k^{*}} \text { Given, } \\
& \rho, \quad \operatorname{Sup}_{H_{0}} \log L_{0}(\rho)=-\left(n_{1}+n_{2}\right) \log 2 \pi-\frac{n_{1}}{2}\left[\log \left(\frac{a_{11.3}}{k}\right)+\log \left(\frac{a_{22.3}}{k}\right)\right] \\
& -\frac{n_{2}}{2}\left[\log \left(\frac{b_{11.3}}{k^{*}}\right)+\log \left(\frac{b_{22.3}}{k^{*}}\right)\right]-\left(\frac{n_{1}+n_{2}}{2}\right) \log \left(i-\rho^{2}\right)-\left(n_{1}+n_{2}\right) \\
& =-\left(n_{1}+n_{2}\right) \log 2 \pi-\frac{n_{1}}{2}\left[\log \left(\frac{a_{11.3}}{n_{1}}\right)+\log \left(\frac{a_{22.3}}{n_{1}}\right)\right]-n_{1} \log \left(1-\rho \hat{\rho}_{12.3}^{(1)}\right)
\end{aligned}
$$




\section{BHANDARY \& GUPTA}

$$
-\frac{n_{2}}{2}\left[\log \left(\frac{b_{11.3}}{n_{2}}\right)+\log \left(\frac{b_{22.3}}{n_{2}}\right)\right]-n_{2} \log \left(1-\rho \hat{\rho}_{12.3}^{(2)}\right)+\left(\frac{n_{1}+n_{2}}{2}\right) \log \left(1-\rho^{2}\right)-\left(n_{1}+n_{2}\right)
$$

(15) is obtained by using (13) and (14).

Now, taking partial derivative of the expression in (15) w.r.t. $\rho$ and setting it to zero, it follows that

$$
\begin{gathered}
-\frac{n_{1}\left(-\hat{\rho}_{12.3}^{(1)}\right)}{1-\rho \hat{\rho}_{12.3}^{(1)}}-\frac{n_{2}\left(-\hat{\rho}_{12.3}^{(2)}\right)}{1-\rho \hat{\rho}_{12.3}^{(2)}}+\frac{\left(n_{1}+n_{2}\right)(-2 \rho)}{2\left(1-\rho^{2}\right)}=0 \\
\Rightarrow \frac{n_{1} \hat{\rho}_{12.3}^{(1)} k}{n_{1}\left(1-\rho^{2}\right)}+\frac{n_{2} \hat{\rho}_{12.3}^{(2)} k^{*}}{n_{2}\left(1-\rho^{2}\right)}-\frac{\left(n_{1}+n_{2}\right) \rho}{1-\rho^{2}}=0(\text { using }(13) \text { and }(14)) \\
\text { i.e., } \hat{\rho}=\frac{k \hat{\rho}_{12.3}^{(1)}+k^{*} \hat{\rho}_{12.3}^{(2)}}{n_{1}+n_{2}} .
\end{gathered}
$$

Note: Asymptotically, $k \simeq n_{1}$ and $k^{*} \simeq n_{2}$ under $H_{0}$.

Hence,

$$
\operatorname{Sup}_{H_{0}} L_{0}=\frac{\left(1-\hat{\rho}^{2}\right)^{\frac{n_{1}+n_{2}}{2}}}{(2 \pi)^{n_{1}+n_{2}}\left(\frac{a_{11.3}}{n_{1}}\right)^{\frac{n_{1}}{2}}\left(\frac{a_{22.3}}{n_{1}}\right)^{\frac{n_{1}}{2}}\left(1-\hat{\rho} \hat{\rho}_{12.3}^{(1)}\right)^{n_{1}}\left(\frac{b_{11.3}}{n_{2}}\right)^{\frac{n_{2}}{2}}\left(\frac{b_{22.3}}{n_{2}}\right)^{\frac{n_{2}}{2}}\left(1-\hat{\rho} \hat{\rho}_{12.3}^{(2)}\right)^{n_{2}}} \cdot e^{-\left(n_{1}+n_{2}\right)}
$$

Using (7) and (17), likelihood ratio test statistic for testing $H_{0}$ Vs. $H_{1}$ is obtained as follows:

$$
\Lambda=\frac{\left(1-\hat{\rho}^{2}\right)^{\frac{n_{1}+n_{2}}{2}}\left(1-\hat{\rho}_{12.3}^{(1) 2}\right)^{\frac{n_{1}}{2}}\left(1-\hat{\rho}_{12.3}^{(2) 2}\right)^{\frac{n_{2}}{2}}}{\left(1-\hat{\rho} \hat{\rho}_{12.3}^{(1)}\right)^{n_{1}}\left(1-\hat{\rho} \hat{\rho}_{12.3}^{(2)}\right)^{n_{2}}}
$$

where $\hat{\rho}$ is given by (16), $\hat{\rho}_{12.3}^{(1)}$ and $\hat{\rho}_{12.3}^{(2)}$ are given by (7). 


\section{Asymptotic distribution of $\Lambda$ :}

Lemma 1. Suppose that $A, A_{n}, n=1,2, \ldots$ are all $p \times p$ symmetric matrices such that $A_{n}-A=O\left(\alpha_{n}\right)$ and $\alpha_{n} \rightarrow 0$ as $n \rightarrow \infty$. Denote by $g\left(A_{n}\right)$ and $g(A)$ as real valued continuous function of $A_{n}$ and $A$ respectively. Then we have $g\left(A_{n}\right)$ $g(A)=O\left(\alpha_{n}\right)$ as $n \rightarrow \infty$.

Proof: $\quad$ The proof of Lemma 1 can be done the same way as in Zhao, Krishnaiah and Bai (1986).

Lemma 2. Let $\underset{\sim}{X}, \underset{\sim}{X}, \ldots, \underset{\sim}{X}$ be i.i.d. $\sim N_{3}(\underset{\sim}{\mu}, \Sigma)$ where, $\underset{\sim}{\mu}=\left(\mu_{1} \mu_{2} \mu_{3}\right)^{\prime}$ and $\sum=\left(\begin{array}{lll}\sigma_{11} & \sigma_{12} & \sigma_{13} \\ \sigma_{21} & \sigma_{22} & \sigma_{23} \\ \sigma_{31} & \sigma_{32} & \sigma_{33}\end{array}\right)$. Then $S \stackrel{\text { a.s }}{\longrightarrow} \sum$ where $S=\frac{1}{n} \sum_{i=1}^{2}\left(\underset{\sim i}{X_{i}}-\bar{X}\right)\left(\underset{\sim}{X_{i}}-\bar{X}\right)^{\prime}$

Proof: $\quad$ Proof of Lemma 2 follows from the Strong Law of Large Numbers.

Lemma 3. Let $\rho_{12.3}$ be the partial correlation coefficient between $X_{1}$ and $X_{2}$, given $X_{3}=x_{3}$. It can be written that $\rho_{12.3}=\frac{\sigma_{12.3}}{\sqrt{\sigma_{11.3}} \sqrt{\sigma_{22.3}}}=\frac{\operatorname{cov}\left(x_{1.3}, x_{2.3}\right)}{\sqrt{\operatorname{var}\left(x_{1.3}\right) \operatorname{var}\left(x_{2.3}\right)}}$ i.e., $\rho_{12.3}$ is a continuous function of $\Sigma$. Let $\quad \hat{\rho}_{12.3}=$ estimate of $\rho_{12.3}=\frac{s_{12.3}}{\sqrt{s_{11.3}} \sqrt{s_{22.3}}}$, where $\quad S_{1.2}=\left(\begin{array}{ll}s_{11} & s_{12} \\ s_{21} & s_{22}\end{array}\right)-\frac{1}{s_{33}}\left(\begin{array}{l}s_{13} \\ s_{23}\end{array}\right)\left(\begin{array}{ll}s_{12} & s_{23}\end{array}\right)=\left(\begin{array}{ll}s_{11.3} & s_{12.3} \\ s_{21.3} & s_{22.3}\end{array}\right)$ i.e., $\quad \hat{\rho}_{12.3} \quad$ is a continuous function of $S$. Then $\hat{\rho}_{12.3} \stackrel{\text { a.s. }}{\longrightarrow} \rho_{12.3}$.

Proof: $\quad$ Since $\hat{\rho}_{12.3}$ is a continuous function of $S$ and $\rho_{12.3}$ is a continuous function of $\Sigma$, the proof of Lemma 3 follows easily from Lemma 1 and Lemma 2.

Theorem 1: Let $\Lambda$ be the likelihood ratio test statistic given by (18) for testing $H_{0}$ vs. $H_{1}$. Then $-2 \log \Lambda \sim \chi_{1}^{2}$ under $H_{0}$ as $n_{1}, n_{2} \rightarrow \infty$, where the symbol $\chi_{1}^{2}$ denotes chi-square distribution with 1 degree of freedom. 


\section{BHANDARY \& GUPTA}

Proof: $\quad$ Using Lemma 3, it follows under $H_{0}$ that,

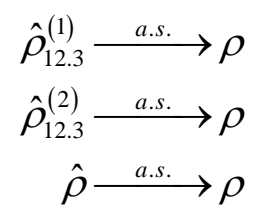

where, $\hat{\rho}_{12.3}^{(1)}, \hat{\rho}_{12.3}^{(2)}, \hat{\rho}$ and $\rho$ are given by (7), (8) and (16). Now, the expression of $\Lambda$ in (18) is asymptotically equivalent to

$$
\Lambda=\frac{\left(1-\hat{\rho}^{2}\right)^{\frac{n_{1}+n_{2}}{2}}\left(1-\rho^{2}\right)^{\frac{n_{1}}{2}}\left(1-\rho^{2}\right)^{\frac{n_{2}}{2}}}{\left(1-\rho^{2}\right)^{n_{1}}\left(1-\rho^{2}\right)^{n_{2}}}(\operatorname{using}(19))=\frac{\left(1-\hat{\rho}^{2}\right)^{\frac{n_{1}+n_{2}}{2}}}{\left(1-\rho^{2}\right)^{\frac{n_{1}+n_{2}}{2}}} .
$$

Hence, $-2 \log \Lambda=-n\left[\log \left(1-\hat{\rho}^{2}\right)-\log \left(1-\rho^{2}\right)\right]$, where $n=n_{1}+n_{2}$

$=-n[\log (1-\hat{\rho})+\log (1+\hat{\rho})-\log (1-\rho)-\log (1+\rho)]$

$=-n[\{\log (1-\hat{\rho})-\log (1-\rho)\}+\{\log (1+\hat{\rho})-\log (1+\rho)\}]$

$=-n\left[\begin{array}{l}\left\{(\rho-\hat{\rho})\left(-\frac{1}{1-\rho}\right)+\frac{(\rho-\hat{\rho})^{2}}{2 !}\left(-\frac{1}{(1-\rho)^{2}}\right)-O\left(\alpha_{n}\right)\right\} \\ +\left\{(\hat{\rho}-\rho)\left(\frac{1}{1+\rho}\right)+\frac{(\hat{\rho}-\rho)^{2}}{2 !}\left(-\frac{1}{(1+\rho)^{2}}\right)+O\left(\alpha_{n}\right)\right\}\end{array}\right]$

(by Taylor Series expansion and $\alpha_{n} \rightarrow 0$ as $n \rightarrow \infty$ )

$$
\begin{aligned}
& =-n\left[\frac{2(\hat{\rho}-\rho)}{1-\rho^{2}}-(\hat{\rho}-\rho)^{2} \frac{\left(1+\rho^{2}\right)}{\left(1-\rho^{2}\right)^{2}}\right] \text { as } n \rightarrow \infty \\
& =-n O\left(\alpha_{n}\right)+\frac{n(\hat{\rho}-\rho)^{2}}{\left(1-\rho^{2}\right)^{2}}+\frac{n(\hat{\rho}-\rho)^{2} \rho^{2}}{\left(1-\rho^{2}\right)^{2}} \\
& =-n O\left(\alpha_{n}\right)+\frac{(\hat{\rho}-\rho)^{2}}{\frac{\left(1-\rho^{2}\right)^{2}}{n}}+n O\left(\alpha_{n}\right)
\end{aligned}
$$


where $\alpha_{n} \rightarrow 0$ as $n \rightarrow \infty$ (using (19)). Since $\frac{\sqrt{n}(\hat{\rho}-\rho)}{1-\rho^{2}} \stackrel{L}{\longrightarrow} N(0,1)$ as $n \rightarrow \infty$ (Anderson (2003), p.133), it is obvious that

$$
\frac{(\hat{\rho}-\rho)^{2}}{\frac{\left(1-\rho^{2}\right)^{2}}{n}} \stackrel{L}{\longrightarrow} \chi_{1}^{2} \text { as, } n \rightarrow \infty \text { where } \stackrel{L}{\longrightarrow} \text { denotes convergence in distribution. (21) }
$$

Theorem 1 follows from (20) and (21).

\section{Large sample Z-test:}

Under this case of $p=3$, it can be shown that (Anderson (2003)) for large sample sizes, $z_{1} \sim N\left(\varsigma_{1}, \frac{1}{n_{1}-4}\right)$ and $z_{2} \sim N\left(\varsigma_{2}, \frac{1}{n_{2}-4}\right)$

where, $z_{1}=\frac{1}{2} \log \frac{1+\hat{\rho}_{12.3}^{(1)}}{1-\hat{\rho}_{12.3}^{(1)}}, \varsigma_{1}=\frac{1}{2} \log \frac{1+\rho_{12.3}^{(1)}}{1-\rho_{12.3}^{(1)}}$

$$
\text { and } z_{2}=\frac{1}{2} \log \frac{1+\hat{\rho}_{12.3}^{(2)}}{1-\hat{\rho}_{12.3}^{(2)}}, \varsigma_{2}=\frac{1}{2} \log \frac{1+\rho_{12.3}^{(2)}}{1-\rho_{12.3}^{(2)}}
$$

The following large sample Z-test for testing $H_{0}$ Vs. $H_{1}$ is proposed:

$$
Z=\frac{\left|z_{1}-z_{2}\right|}{\sqrt{\frac{1}{n_{1}-4}+\frac{1}{n_{1}-4}}}>1.96 \text { at } 5 \% \text { level of significance. }
$$

The two tests given by (18) and (22) are compared by power analysis in the next section.

\section{Simulation Results}

Multivariate normal random vectors using $\mathrm{R}$ program are generated in order to evaluate the power and size of the two tests given by (18) and (22). The $R$ program produced estimates of $\rho_{12.3}^{(1)}, \rho_{12.3}^{(2)}$ and $\rho$ (given by (7) and (16)) along with the Approximate Likelihood Ratio (ALR) statistic given by (18) and the 


\section{BHANDARY \& GUPTA}

Z-statistic given by (22) 5,000 times for each particular combination of population parameters $\left(\rho_{12.3}^{(1)}\right.$ and $\left.\rho_{12.3}^{(2)}\right)$. The frequency of rejection of each test statistic at $\alpha=0.05$ was noted and the proportion of rejections (power) are reported in Table 1 for various combinations of $\rho_{1}$ and $\rho_{2}\left(\rho_{12.3}^{(1)}\right.$ and $\left.\rho_{12.3}^{(2)}\right)$.

On the basis of our study, it is found that the ALR-test showed consistently better results than $\mathrm{Z}$ - test in terms of power. The size of the ALR test is slightly more than alpha level. The ALR test is recommended strongly for use in practice

Table 1. Empirical significance level and power of the Approximate Likelihood Ratio (APR) test and the Z-test (ZT) for $p=3$ and $\alpha=0.05$

\begin{tabular}{rrrrrrrrrrr}
\multicolumn{10}{c}{$N_{1}=N_{2}=25$} \\
\hline$\rho_{1}$ & $\rho_{2}$ & 0.1 & 0.2 & 0.3 & 0.4 & 0.5 & 0.6 & 0.7 & 0.8 & 0.9 \\
\hline 0.1 & ALR & 0.050 & 0.067 & 0.107 & 0.187 & 0.307 & 0.453 & 0.639 & 0.758 & 0.998 \\
& ZT & 0.050 & 0.054 & 0.068 & 0.101 & 0.158 & 0.259 & 0.431 & 0.684 & 0.945 \\
0.3 & ALR & 0.100 & 0.062 & 0.054 & 0.069 & 0.125 & 0.242 & 0.414 & 0.640 & 0.981 \\
& ZT & 0.069 & 0.055 & 0.049 & 0.057 & 0.086 & 0.148 & 0.280 & 0.530 & 0.884 \\
0.5 & ALR & 0.300 & 0.195 & 0.127 & 0.074 & 0.053 & 0.081 & 0.178 & 0.420 & 0.727 \\
& ZT & 0.159 & 0.119 & 0.085 & 0.061 & 0.051 & 0.066 & 0.130 & 0.319 & 0.743 \\
0.7 & ALR & 0.621 & 0.528 & 0.426 & 0.297 & 0.184 & 0.089 & 0.052 & 0.118 & 0.472 \\
& ZT & 0.425 & 0.354 & 0.279 & 0.202 & 0.131 & 0.076 & 0.050 & 0.101 & 0.428 \\
0.9 & ALR & 0.998 & 0.995 & 0.981 & 0.956 & 0.902 & 0.782 & 0.586 & 0.282 & 0.063 \\
& ZT & 0.945 & 0.920 & 0.884 & 0.828 & 0.742 & 0.613 & 0.429 & 0.202 & 0.050 \\
\hline & & & & $N_{1}=25, N_{2}=50$ & & & & \\
\hline 0.1 & ALR & 0.059 & 0.077 & 0.143 & 0.263 & 0.469 & 0.670 & 0.876 & 0.977 & 0.999 \\
& ZT & 0.050 & 0.056 & 0.076 & 0.119 & 0.202 & 0.338 & 0.552 & 0.814 & 0.986 \\
0.3 & ALR & 0.122 & 0.065 & 0.052 & 0.065 & 0.145 & 0.284 & 0.489 & 0.721 & 0.802 \\
& ZT & 0.076 & 0.058 & 0.049 & 0.060 & 0.099 & 0.186 & 0.364 & 0.661 & 0.959 \\
0.5 & ALR & 0.466 & 0.305 & 0.186 & 0.095 & 0.059 & 0.010 & 0.257 & 0.610 & 0.965 \\
& ZT & 0.200 & 0.145 & 0.098 & 0.064 & 0.050 & 0.072 & 0.163 & 0.414 & 0.863 \\
0.7 & ALR & 0.881 & 0.782 & 0.633 & 0.453 & 0.279 & 0.128 & 0.066 & 0.166 & 0.692 \\
& ZT & 0.548 & 0.461 & 0.363 & 0.261 & 0.164 & 0.085 & 0.050 & 0.120 & 0.551 \\
0.9 & ALR & 1.000 & 0.999 & 0.997 & 0.992 & 0.960 & 0.885 & 0.707 & 0.356 & 0.069 \\
& ZT & 0.987 & 0.977 & 0.958 & 0.925 & 0.864 & 0.750 & 0.550 & 0.261 & 0.050 \\
\hline & & & & & & & & & &
\end{tabular}


TWO POPULATION PARTIAL CORRELATION COEFFICIENT TEST

\section{Acknowledgement}

Authors would like to thank Mr. Mohammad Aziz for computational help.

\section{References}

Anderson, T. W. (2003). An introduction to multivariate statistical analysis (3rd ed.). Hoboken, N.J: John Wiley \& Sons.

Fisher, R. A. (1924). The distribution of the partial correlation coefficient. Metron, 3, 329-332.

Gupta, A. K. and Kabe, D. G. (2001). On testing the equality of K multiple and partial correlation coefficients. ACTA Mathematica Scientia, 21(2), 221-223.

Zhao, L. C., Krishnaiah, P. R. \& Bai, Z. D. (1986). On detection of number of signals in presence of white noise. Journal of Multivariate Analysis, 20(1), 1-25. doi:10.1016/0047-259X(86)90017-5 